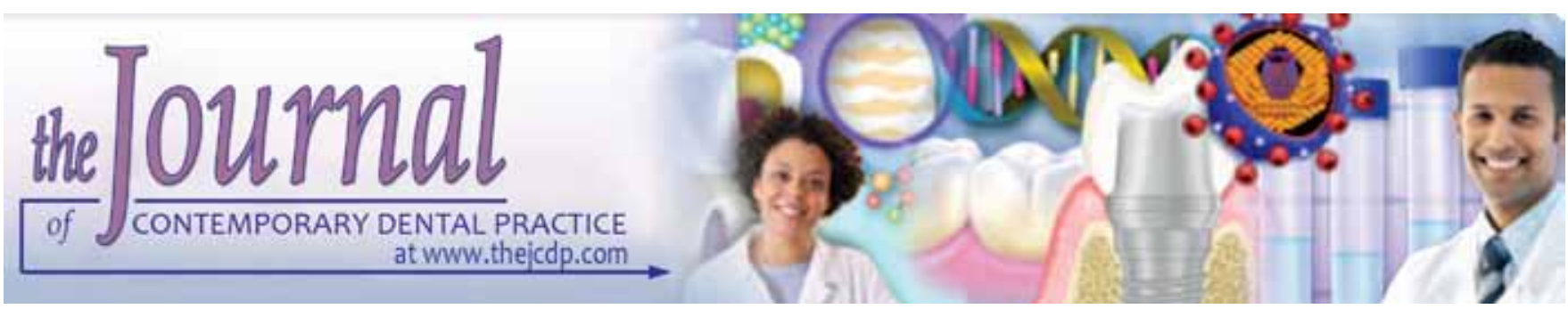

\title{
A Comparative Study of Post-traumatic Stress Disorder, Anxiety, Depression and Change in Sexual Functioning of Patients with Facial Injuries and Patients with Extremity Injuries
}

\author{
${ }^{1}$ NT Prashanth, ${ }^{2}$ HP Raghuveer, ${ }^{3}$ Dilip Kumar, ${ }^{4}$ ES Shobha, ${ }^{5}$ Vinod Rangan, ${ }^{6}$ TSS Rao
}

\begin{abstract}
Background: This study was performed to identify and compare the presence of and the levels of post-traumatic stress disorder (PTSD), anxiety, depression and if any change in sexual functioning is present in patients with disfiguring facial injuries, nondisfiguring facial injuries and visible extremity injuries.
\end{abstract}

Materials and methods: A total of 378 patients from multi modal trauma centers in Bengaluru city were included for the study. A total of 153 patients were those who had disfiguring facial injuries, 111 patients with nondisfiguring facial injuries and 114 patients had sustained a visible extremity injury that was not covered by clothing (disfigurement/scarring). The assessments were carried out at three time intervals (the date of discharge, 1 month postoperatively and 6 months postoperatively) of the follow-up.

The impact of events scale (IES), the hospital anxiety and depression scale (HADS) and changes in sexual functioning questionnaire (CSFQ) were used.

Results: Statistically significant higher means of IES, HADS (both for anxiety and depression) were present in patients with disfiguring facial injuries compared to patients with nondisfiguring facial injuries and visible extremity injuries and this was present at all three study intervals (date of discharge,1st postoperative month and 6th postoperative month). There was a statistically significant change in the means of CSFQ scores of visible extremity injuries compared to other groups at the date of discharge bordering on sexual dysfunction, there was a raise

\footnotetext{
${ }^{1-5}$ Department of Oral and Maxillofacial Surgery, Dayananda Sagar College of Dental Sciences, Bengaluru, Karnataka, India

${ }^{6}$ Department of Psychiatry, JSS Medical College, Mysore Karnataka, India

Corresponding Author: NT Prashanth, Associate Professor Department of Oral and Maxillofacial Surgery, Dayananda Sagar College of Dental Sciences, SM Hills, Kumaraswamy Layout, Bengaluru-560 078, Karnataka, India, e-mail: dr.n.t.prashanth@gmail.com
}

in CSFQ scores among all three groups at the 1st postoperative month and the 6th postoperative month which was above the dysfunction cut-off.

Conclusion: The results of this study led to the conclusion that in comparison with patients who had sustained disfiguring facial injuries, nondisfiguring facial injuries and visible extremity/ orthopedic injuries, the mean IES scores, HADS scores for both anxiety and depression of the facial disfiguring injury patients was higher at all three study intervals (date of discharge,1st postoperative month and 6 months postoperatively), indicating higher PTSD levels, anxiety and depression in patients with disfiguring facial injuries, CSFQ scores were above the cutoff suggested at the 1st month and 6th month postoperative indicating no sexual dysfunction in all the three groups.

Keywords: Anxiety, Depression, Facial injuries, Maxillofacial injuries, Post-traumatic stress disorder.

How to cite this article: Prashanth NT, Raghuveer HP, Kumar D, Shobha ES, Rangan V, Rao TSS. A Comparative Study of Posttraumatic Stress Disorder, Anxiety, Depression and Change in Sexual Functioning of Patients with Facial Injuries and Patients with Extremity Injuries. J Contemp Dent Pract 2015;16(11): 884-892.

\section{Source of support: Nil}

Conflict of interest: None

\section{INTRODUCTION}

The face plays an important part in the formation of initial social relationships ${ }^{1}$ and the appearance or 'attractiveness' of a person is greatly contributed by the face.

Trauma is a major cause for disfigurement of the face while there are many secondary causes too, extensive hard tissue and soft tissue injuries can lead to scarring/ disfigurement.

Psychological distress is common sequelae of such trauma; there has been significant progress in the care of facial trauma in the hospital setting as recent research has focused on the psychological aspects of the trauma. 
Military veterans and disaster survivors are our primary knowledge of the psychological impacts of the traumatic events. ${ }^{2}$ Facial trauma was recognized as important for research because of its potential for both physical and psychological disability, ${ }^{3}$ nonrecognition or under recognition and nontreatment of this important morbidity following maxillofacial trauma is further compounded by poor documentation in routine clinical practice, an untreated psychological impact can become chronic.

There has been published literature suggesting anxiety, depression and post-traumatic stress disorder (PTSD) can develop following facial injuries ${ }^{4,5}$ and that PTSD in facial trauma patients range between 26 and $41 \% .{ }^{6,7}$

Anxiety is an emotion characterized by a state of unpleasantness and of inner turmoil, it is accompanied by nervous behavior, such as pacing back and forth, somatic complaints and rumination. ${ }^{8}$ There are subjective unpleasant feelings of dread over anticipated events, such as the feeling of imminent death. ${ }^{9}$ Anxiety is not the same as fear that is a response to a real or perceived immediate threat whereas anxiety is the expectation of future threat. ${ }^{10}$ Anxiety is a feeling of fear, worry, and uneasiness, usually generalized and unfocused as an overreaction to a situation that is only subjectively seen as menacing. There is muscular tension, restlessness, fatigue and problems in concentration. Anxiety can be appropriate, but when experienced regularly the individual may suffer from an anxiety disorder.

Depression is a state of low mood and aversion to activity that can affect a person's thoughts, behavior, feelings and sense of well-being. ${ }^{11,12}$ People with depressed mood can feel sad, anxious, empty, hopeless, helpless, worthless, guilty, irritable, ashamed or restless. They may lose interest in activities that were once pleasurable, experience loss of appetite or overeating, have problems concentrating, remembering details or making decisions, and may contemplate, attempt or commit suicide. Insomnia, excessive sleeping, fatigue, aches, pains, digestive problems or reduced energy may also be present. ${ }^{13}$

Psychiatric illness especially anxiety disorders and depression can have a negative impact on sexual functioning ${ }^{14}$ so the presence of anxiety and depression in patients with facial and extremity injuries and its correlation if any with sexual functioning of the patient was investigated.

The aim of this study was to identify the presence and compare anxiety, depression and change in sexual functioning in patients who had facial injuries (nondisfiguring, disfiguring) and patients who had disfiguring traumatic injuries or scars on their extremities that is visible and not covered by clothing.

The inclusion criteria for the patients in the study are as follows:

- Age - 18 years or older

- Glasgow coma scale on admission of 12 and above

- Patients with facial injuries leading to scarring of $3 \mathrm{~cm}$ or more

- Patients with facial injuries leading to facial disfigurement/asymmetry post-treatment.

- Patients who had sustained nondisfiguring facial injuries

- Patients who had a visible injury (disfigurement/ scarring) on their extremities.

The exclusion criteria are as follows:

- Patients with a history of alcohol dependence.

- Patients with past history of psychological disturbances.

The participants were 378 patients from multi modal trauma centers in Bengaluru city who were included for the study. Of these, 153 patients were those who had facial injuries among them 81 patients were male (51 less than 50 years of age and 30 more than 50 years of age) and 72 patients were female (40 less than 50 years of age and 32 more than 50 years of age) and 111 patients with nondisfiguring facial injuries out of which 54 were male patients and 57 were female patients. A total of 114 patients had sustained a visible extremity injury that was not covered by clothing (disfigurement/scarring) and among them 51 were male patients and 63 were female patients.

The assessments were carried out at three time intervals (the date of discharge, 1 month postoperatively and 6 months postoperatively) of the follow-up.

The impact of event scale (IES) 15 was used to assess the PTSD; this is a widely used valid and reliable scale and provides for a low-cost short self-report measurement to detect PTSD. ${ }^{16}$

The IES scale consists of 15 items as 7 of which measure intrusive symptoms (intrusive thoughts, nightmares, intrusive feelings and imagery) and 8 of which measure avoidance symptoms (numbing of responsiveness, avoidance of feelings, situations and ideas) and both combined provide a subjective stress score. The responders to the scale rate the items on a fourpoint scale according to how often each of them occurred in the past 7 days. ${ }^{17}$

\section{Score Consequence}

Twenty-seven or more: There is a $75 \%$ chance that the patient has PTSD $^{18}$ and those who do not have full PTSD may have partial PTSD or at least some of the symptoms. 
Thirty-five and above: This represents the bestcut-off for a probable diagnosis of PTSD - a mental health professional skilled to treat such issues should be consulted. ${ }^{19}$

The hospital anxiety and depression scale (HADS) was used to assess the anxiety and depression of the facial trauma patients. It is a widely used, valid and reliable scale and provides for a low cost short self report measurement to detect anxiety and depression.

Hospital anxiety and depression scale was originally developed by Zigmond and Snaith (1983) and is commonly used by doctors to determine the levels of anxiety and depression that a patient is experiencing. ${ }^{20}$ The HADS is a fourteen item scale that generates ordinal data. Seven of the items relate to anxiety and seven relate to depression.

The changes in sexual functioning questionnaire (male and female) is a structured interview/questionnaire designed to measure illness and medication related changes in sexual functioning. It takes into account five domains of sexual functioning pleasure, desire/ frequency, desire/interest, arousal/excitement and orgasm/completion.

\section{RESULTS}

\section{Statistical Analysis}

The statistical analysis was done by Mann-Whitney U test for determining and comparing the IES, the HADS and CSFQ scores among the patients and the following results were obtained (Table 1).

On comparison of IES scores among patients with disfiguring facial injuries, nondisfiguring facial injuries and orthopedic/visible scarring(extremity injuries) at the date of discharge the mean IES score of disfiguring facial injury patients was $48.39 \pm 10.29$ while the patients with nondisfiguring facial injuries it was $32.05 \pm 7.89$ and for the orthopedic/visible injury patients it was $9.45 \pm 4.32$. There is a statistically significant difference in the mean scores of disfiguring facial injury patients compared to nondisfiguring facial injury patients and visible extremity injury patients.

At the 1st month postoperative period the mean IES scores of patients with facial disfiguring injuries was 35.16 \pm 12.08 , for the nondisfiguring facial injury patients the mean IES was $6.17 \pm 2.17$ and for the visible extremity injury patients the mean IES was $2.84 \pm 5.76$. Statistically, significant difference is observed in disfiguring facial injury patients compared to nondisfiguring facial injury patients and visible extremity injury patients.

At the 6th postoperative month period, the mean IES of disfiguring facial injury patients was $29.02 \pm 13.49$, the mean IES of nondisfiguring facial injury patients was $2.07 \pm 0.37$ and for visible extremity injury patients it was $1.68 \pm 3.31$. Statistically significant difference is observed in disfiguring facial injury patients compared to nondisfiguring facial injury patients and visible extremity injury patients.

On comparison of HADS for the anxiety components among patients with disfiguring facial injuries, nondisfiguring facial injuries and orthopedic/visible scarring (extremity injuries) at the date of discharge the mean HADS score of disfiguring facial injury patients was $16.34 \pm 3.28$ while the patients with nondisfiguring facial injuries it was $8.10 \pm 2.51$ and for the orthopedic/visible injury patients it was $5.86 \pm 2.51$. There is a statistically significant difference in the mean scores of disfiguring facial injury patients compared to nondisfiguring facial injury patients and visible extremity injury patients.

At the 1st month postoperative period the mean HADS scores of patients with facial disfiguring injuries was $13.76 \pm 2.90$, for the nondisfiguring facial injury patients the mean HADS was $3.48 \pm 1.99$ and for the visible extremity injury patients the mean HADS was 2.09 \pm 2.93 . Statistically significant difference is observed in the means of disfiguring facial injury patients compared to nondisfiguring facial injury patients and visible extremity injury patients.

At the 6th postoperative month period, the mean HADS of disfiguring facial injury patients was $12.16 \pm$ 2.28 , the mean HADS of nondisfiguring facial injury patients was $1.36 \pm 1.40$ and for visible extremity injury patients it was $1.11 \pm 2.15$. Statistically, significant difference is observed in the means of disfiguring facial injury patients compared to nondisfiguring facial injury patients and visible extremity injury patients.

On comparison of HADS for the depression components among patients with disfiguring facial injuries, nondisfiguring facial injuries and orthopedic/ visible scarring (extremity injuries) at the date of discharge the mean HADS score of disfiguring facial injury patients was $15.90 \pm 3.53$ while the patients with nondisfiguring facial injuries it was $8.84 \pm 2.51$ and for the orthopedic/visible injury patients it was $3.86 \pm 2.63$. There is a statistically significant difference in the mean scores of disfiguring facial injury patients compared to nondisfiguring facial injury patients and visible extremity injury patients.

At the 1st month postoperative period, the mean HADS scores of patients with facial disfiguring injuries was $13.01 \pm 2.47$, for the nondisfiguring facial injury patients the mean HADS was $3.68 \pm 2.00$ and for the visible extremity injury patients the mean HADS was 2.41 \pm 2.60 . Statistically, significant difference is observed in the means of disfiguring facial injury patients compared 
Table 1: Comparison of all three types of injuries (entire sample)

\begin{tabular}{|c|c|c|c|c|c|c|c|c|}
\hline Scale & Time interval & Injury & $n$ & Mean & Std. dev. & Min. & Max. & $p$-value \\
\hline \multirow[t]{9}{*}{ Impact of events } & DOD & Nondisfiguring facial injury & 111 & 32.05 & 7.87 & 13.00 & 40 & $<0.001^{*}$ \\
\hline & & Disfiguring facial injury & 153 & 48.39 & 10.29 & 36.00 & 66 & \\
\hline & & Visible extremity injury & 114 & 9.45 & 4.32 & 6.00 & 21 & \\
\hline & 1 Month & Nondisfiguring facial injury & 111 & 6.17 & 2.17 & 2.00 & 11 & $<0.001^{*}$ \\
\hline & & Disfiguring facial injury & 153 & 35.16 & 12.08 & 15.00 & 55 & \\
\hline & & Visible extremity injury & 114 & 2.84 & 5.76 & 0.00 & 17 & \\
\hline & 6 Months & Nondisfiguring facial injury & 111 & 2.07 & 0.37 & 1.00 & 3 & $<0.001^{*}$ \\
\hline & & Disfiguring facial injury & 153 & 29.02 & 13.49 & 10.00 & 53 & \\
\hline & & Visible extremity injury & 114 & 1.68 & 3.31 & 0.00 & 9 & \\
\hline \multirow[t]{9}{*}{ CSFQ } & DOD & Nondisfiguring facial injury & 111 & 49.72 & 6.18 & 20.00 & 56 & $<0.001^{*}$ \\
\hline & & Disfiguring facial injury & 153 & 45.73 & 3.09 & 42.00 & 56 & \\
\hline & & Visible extremity injury & 114 & 43.39 & 7.90 & 26.00 & 52 & \\
\hline & 1 Month & Nondisfiguring facial injury & 111 & 54.14 & 6.72 & 23.00 & 63 & $<0.001^{*}$ \\
\hline & & Disfiguring facial injury & 153 & 49.22 & 4.22 & 42.00 & 64 & \\
\hline & & Visible extremity injury & 114 & 48.94 & 8.95 & 28.00 & 57 & \\
\hline & 6 Months & Nondisfiguring facial injury & 111 & 56.57 & 7.77 & 19.00 & 64 & $<0.001^{*}$ \\
\hline & & Disfiguring facial injury & 153 & 50.46 & 5.02 & 42.00 & 66 & \\
\hline & & Visible extremity injury & 114 & 49.73 & 8.39 & 31.00 & 58 & \\
\hline \multirow[t]{9}{*}{ HADS anxiety } & DOD & Nondisfiguring facial injury & 111 & 8.10 & 2.51 & 4.00 & 11 & $<0.001^{*}$ \\
\hline & & Disfiguring facial injury & 153 & 16.34 & 3.28 & 8.00 & 18 & \\
\hline & & Visible extremity injury & 114 & 5.86 & 2.51 & 4.00 & 11 & \\
\hline & 1 Month & Nondisfiguring facial injury & 111 & 3.48 & 1.99 & 1.00 & 6 & $<0.001^{*}$ \\
\hline & & Disfiguring facial injury & 153 & 13.76 & 2.90 & 6.00 & 17 & \\
\hline & & Visible extremity injury & 114 & 2.09 & 2.93 & 0.00 & 8 & \\
\hline & 6 Months & Nondisfiguring facial injury & 111 & 1.36 & 1.40 & 0.00 & 3 & $<0.001^{*}$ \\
\hline & & Disfiguring facial injury & 153 & 12.16 & 2.28 & 6.00 & 15 & \\
\hline & & Visible extremity injury & 114 & 1.11 & 2.15 & 0.00 & 6 & \\
\hline \multirow[t]{9}{*}{ HADS depression } & DOD & Nondisfiguring facial injury & 111 & 8.84 & 2.51 & 2.00 & 11 & $<0.001^{*}$ \\
\hline & & Disfiguring facial injury & 153 & 15.90 & 3.53 & 8.00 & 18 & \\
\hline & & Visible extremity injury & 114 & 3.86 & 2.63 & 1.00 & 9 & \\
\hline & 1 Month & Nondisfiguring facial injury & 111 & 3.68 & 2.00 & 0.00 & 6 & $<0.001^{*}$ \\
\hline & & Disfiguring facial injury & 153 & 13.01 & 2.47 & 8.00 & 15 & \\
\hline & & Visible extremity injury & 114 & 2.41 & 2.60 & 0.00 & 7 & \\
\hline & 6 Months & Nondisfiguring facial injury & 111 & 1.22 & 1.40 & 0.00 & 3 & $<0.001^{*}$ \\
\hline & & Disfiguring facial injury & 153 & 10.47 & 1.15 & 8.00 & 11 & \\
\hline & & Visible extremity injury & 114 & 1.00 & 2.02 & 0.00 & 6 & \\
\hline
\end{tabular}

*Denotes significant difference

to nondisfiguring facial injury patients and visible extremity injury patients.

At the 6th postoperative month period, the mean HADS of disfiguring facial injury patients was $10.47 \pm$ 1.15 , the mean HADS of nondisfiguring facial injury patients was $1.22 \pm 1.40$ and for visible extremity injury patients it was $1.00 \pm 2.02$. Statistically, significant difference is observed in the means of disfiguring facial injury patients compared to nondisfiguring facial injury patients and visible extremity injury patients.

On comparison of CSFQ Scores among the patients with facial disfiguring injuries, nondisfiguring facial injuries and visible extremity injuries, though the CSFQ cut off scores of males is 47 and for females its 41 , the present study consisted of both male and female patients. At the date of discharge (DOD) the mean CSFQ for the facial disfiguring injury patients the mean CSFQ was $45.73 \pm 3.09$, for nondisfiguring facial injury patients the mean CSFQ was $49.72 \pm 6.18$ and for visible extremity injury patients the mean CSFQ was $43.39 \pm 7.90$. Though there is a statistical significance in the means of CSFQ of visible orthopedic/extremity injury patients and others it was not low enough to consider that it met the cut off to denote sexual dysfunction as this was done among a cross section of patients involving both males and females.

The mean CSFQ of patients with disfiguring facial injuries at 1 month postoperatively was $49.22 \pm 4.22$ and for patients with nondisfiguring facial injury it was $54.14 \pm 6.72$ and for visible extremity injury patients it was $48.94 \pm 4.22$.

The mean CSFQ of patients with disfiguring facial injuries at 6 months postoperatively was $50.46 \pm 5.02$ 
and for patients with nondisfiguring facial injury it was $56.57 \pm 7.77$ and for visible extremity injury patients it was $49.73 \pm 8.39$.

\section{DISCUSSION}

A lot of focus in the field of research in trauma is on assessing and predicting the psychological morbidity that follows a facial injury. Earlier research and literature have established the presence of PTSD in patients with maxillofacial injuries. ${ }^{1,4,21,22}$

There has also been published research on anxiety and depression following facial injuries and also the anxiety and depression levels in patients with disfiguring facial injuries and nondisfiguring facial injuries. ${ }^{4}$

Since presence of anxiety and depression can have a negative impact on sexual functioning, there is not much information documenting the change in sexual functioning of patients who had suffered facial injuries and also there is not much information on the comparison of PTSD levels, anxiety and depression levels and the sexual dysfunction levels in patients who suffered disfiguring facial injuries, nondisfiguring facial injuries and visible extremity injuries.

The present study is a comparative evaluation of PTSD levels, levels of anxiety and depression and the change in sexual functioning of patients who have suffered facial disfiguring injuries, nondisfiguring injuries of the face and visible extremity injuries.

This study shows that there is a statistically significant increase of IES scores in patients with disfiguring facial injuries compared to nondisfiguring facial injuries and visible extremity injuries at all study intervals namely DOD, 1st postoperative month and 6 months postoperatively.

The study also found that there were raised IES scores of nondisfiguring facial injury patients compared to visible extremity patients at all three study intervals.

This study shows that there is a statistically significant increase of HADS scores of both anxiety and depression in patients with disfiguring facial injuries compared to nondisfiguring facial injuries and visible extremity injuries at all study intervals namely DOD, 1st postoperative month and 6 months postoperatively.

The study also found that there were raised HADS scores for both anxiety and depression of nondisfiguring facial injury patients compared to visible extremity patients at all three study intervals.

On comparison of CSFQ scores of patients with disfiguring facial injuries, nondisfiguring facial injuries and visible extremity/orthopedic injuries, though there was a statistical difference in the means of CSFQ of patients with visible extremity injuries compared to the others, it was not low enough to the cut off suggested in the scale to denote sexual dysfunction though the cut off is 41 for females and 47 for males in the CSFQ scale. This requires further research among the data collected to arrive at a confirmation. These low scores was only observed at the date of discharge while the mean CSFQ scores of all the subjects increased at the 1st month postoperative period and the 6th month postoperative period and also it was above the cut-off suggested for sexual dysfunction in the CSFQ questionnaire for both males and females.

Our study showed the presence of PTSD, anxiety and depression and also that its levels are significantly more in patients with disfiguring facial injuries compared to nondisfiguring facial injuries and visible extremity injuries. This reiterates that we have to strive to provide psychological counseling and treatment for disfiguring facial injury patients so that the trauma care is comprehensive.

\section{CONCLUSION}

The results of this study led to the conclusion that in comparison with patients who had sustained disfiguring facial injuries, nondisfiguring facial injuries and visible extremity/orthopedic injuries, the mean IES scores of facial disfiguring injury patients was higher than the mean IES scores of patients with nondisfiguring facial injuries and visible extremity injury patients at all three study intervals (DOD, 1st postoperative month and 6 months postoperatively), indicating higher PTSD levels in patients with disfiguring facial injuries. The mean HADS scores for both anxiety and depression in facial disfiguring injury patients was higher than the mean HADS scores of patients with nondisfiguring facial injuries and visible extremity injury patients at all three study intervals (DOD, 1st postoperative month and 6 months postoperatively), indicating higher anxiety and depression levels in patients with disfiguring facial injuries. There was a slight decrease in the CSFQ scores of patients with visible extremity injuries compared to facial disfiguring injury patients and nondisfiguring facial injury patients, the mean CSFQ score bordered on the cut-off mentioned in the CSFQ questionnaire for sexual dysfunction at the DOD, however a raise in the CSFQ scores was present above the cut-off in all the subjects at the first postoperative month and the 6th postoperative month indicating no sexual dysfunction.

\section{CLINICAL SIGNIFICANCE}

This study reinforces that we have to identify the presence of PTSD, anxiety and depression at an early stage 
especially in patients with disfiguring facial injuries. This will lead to the development of comprehensive care as the trauma patient will be treated for the psychological comorbid condition also.

Awareness can be created among the nursing, paramedical and the surgical staff about the recognition of these early signs of PTSD, anxiety and depression of facial injury patients. The facial injury patients can be given structured psychometric questionnaires and if any of the patients show early signs of PTSD, anxiety or depression, treatment of the same can be initiated by skilled professionals trained to handle such conditions. And along with the management of the facial trauma this can lead to better quality of life of the patient.

\section{REFERENCES}

1. Prashanth NT, RaghuveerHP, KumarRD, ShobhaES, Rangan V, Hullale B. Post-traumatic stress disorder in facial injuries: a comparative study. J Contemp Dent Pract 2015 Feb 1; 16(2):118-125.

2. Alexander DA. Human reactions to trauma: their features and management. Greaves I, Porter K, editors. Pre-hospital medicine: the principles and practice of immediate care, Arnold, London 1999.

3. Shepherd JP. Strategies for the study of long-term sequelae of oral and facial injuries. J Oral Maxillofac Surg 1992;50:390-399.

4. Prashanth NT, Raghuveer HP, Kumar D, Shobha ES, Rangan V, Rao TS. Anxiety and depression in facial injuries: a comparative study. J Int Oral Health 2015 Sep; 7(9):94-100.

5. Bisson JI, Shepherd JP, Dhutia M. Psychological sequelae of facial trauma. J Trauma 1997;43:496-500.

6. Hull AM, Lowe T, Devlin M, Finlay P, Koppel D, Stewart AM. Psychological consequences of maxillofacial trauma: a preliminary study. Br J Oral Maxillofac Surg 2003 Oct; 41(5): 317-322.

7. Roccia F, Dell'Acqua A, Angelini G, Berrone S. Maxillofacial trauma and psychiatric sequelae: post-traumatic stress disorder. J Craniofac Surg 2005 May;16(3):355-360.
8. Seligman MEP, Walker EF, Rosenhan DL. Abnormal psychology. 4th ed. New York: W.W. Norton \& Company.

9. Davison, Gerald C. Abnormal psychology. Toronto: Veronica Visentin. 2008; p. 154. ISBN 978-0-470-84072-6.

10. American Psychiatric Association. Diagnostic and Statistical Manual of Mental Disorders. 5th ed. Arlington, VA: American Psychiatric Publishing. 2013; p. 189.ISBN 978-0-89042-555-558.

11. Salmans, Sandra. Depression: Questions You Have - Answers You Need. People's Medical Society. ISBN 978-1-882606-14-6.

12. Diagnostic and statistical manual of mental disorders, 5 th ed. (DSM-5). American Psychiatric Association. 2013.

13. Available at: http://www.nimh.nih.gov/health/topics/ depression/index.shtml (Accessed May 01, 2015)

14. Teusch N, Scherbaum N, Böhme H. Different patterns of sexual dysfunctions associated with psychiatric disorders and psychopharmacological treatment. Pharmacopsychiatrica 1995;28:84-92.

15. Horowitz M, Wilner N, Alvarez W. Impact of events scale: a measure of subjective stress. Psychosom Med 1979;41(3): 209-218.

16. Rothbaum BO, Foa EB, Riggs DS, Murdock TB, Walsh W. A prospective examination of post-traumatic stress disorder in rape victims. J Traumatic Stress 1992;5:455-475.

17. Devilly GJ, Spence SH. The relative efficacy and treatment distress of EMDR and a cognitive-behaviour trauma treatment protocol in the amelioration of post-traumatic stress disorder. J Anxiety Disorders 1999;13:131-157.

18. Coffey SF, Berglind G, Screening for PTSD in motor vehicle accident survivors using PSS-SR and IES. J Traumatic Stress 2006;19(1):119-128.

19. Neal LA, Walter B, Rollins J, et al. Convergent validity of measures of post-traumatic stress disorder in a mixed military and civilian population. J Traumatic Stress 1994;7(3):447-455.

20. Zigmond AS, Snaith RP. The hospital anxiety and depression scale. Acta Psychiatr Scand 1983 Jun;67(6):361-370.

21. Sen P, Ross N, Rogers S. Recovering maxillofacial trauma patients: the hidden problems. J Wound Care 2001 Mar; 10(3):53-57.

22. Hull AM, Lowe T, Finlay PM. The psychological impact of maxillofacial trauma: an overview of reactions to trauma. Oral Surg Oral Med Oral Pathol Oral Radiol Endod 2003;95(5): 515-520. 


\section{Annexures and Questionnaires}

\section{The IES Questionnaire}

Particulars

a. I thought about it when I did not mean to

b. I avoided letting myself get upset when I thought about it or was reminded of it

c. I tried to remove it from memory

d. I had trouble falling asleep or staying asleep, because of pictures or thoughts about it that came into my mind

e. I had waves of strong feelings about it

f. I had dreams about it

g. I stayed away from reminders of it

h. I felt as if it had not happened or it was not real

i. I tried not to talk about it

j. Pictures about it popped into my mind

k. Other things kept making me think about it

1. I was aware that I still had a lot of feelings about it, but I did not deal with them

$\mathrm{m}$. I tried not to think about it

n. Any reminder brought back feelings about it

o. My feelings about it were kind of number

For each question score 0 for 'not at all', 1 for 'rarely', 3 for 'sometimes' and 5 for 'often' Intrusion total comes from adding the scores for questions a, d, e, f, j, k, n; avoidance total from adding scores for questions $\mathrm{b}, \mathrm{c}, \mathrm{g}, \mathrm{h}, \mathrm{i}, \mathrm{l}, \mathrm{m}, \mathrm{o}$. Add intrusion and avoidance for the full total

Intrusion total $=$

Avoidance total $=$

\section{IES Interpretation}

- 0-8: No meaningful impact.

- 9-25: Impact event - you may be affected.

- 26-43: Powerful impact event - you are certainly affected.

- 44-75: Severe impact event - this is capable of altering your ability of function.

\section{HADS Questionnaire}

The items on the questionnaire that relate to anxiety are as follows:

- I feel tense or wound up

- I get a sort of frightened feeling as if something bad is about to happen

- Worrying thoughts go through my mind

- I can sit at ease and feel relaxed

- I get a sort of frightened feeling like butterflies in the stomach

- I feel restless and have to be on the move

- I get sudden feelings of panic
The items that relate to depression are as follows:

- I still enjoy the things I used to enjoy

- I can laugh and see the funny side of things

- I feel cheerful

- I feel as if I am slowed down

- I have lost interest in my appearance

- I look forward with enjoyment to things

- I can enjoy a good book or radio or TV program

Each item on the questionnaire is scored from 0-3 and this means that a person can score between 0 and 21 for either anxiety or depression. The HADS uses a scale and therefore the data returned from the HADS is ordinal.

Score consequence

$0-7=$ Normal

$8-10=$ Borderline abnormal

\section{Changes in Sexual Functioning Questionnaire for Males: (CSFQ-M-C)}

1. Compared with the most enjoyable it has ever been, how enjoyable or pleasurable is your sexual life right now?

- 1-No enjoyment or pleasure

- 2-Little enjoyment or pleasure

- 3-Some enjoyment or pleasure

- 4-Much enjoyment or pleasure

- 5-Great enjoyment or pleasure

2. How frequently do you engage in sexual activity (sexual intercourse, masturbation, etc.) now?

- 1-Never

- 2-Rarely (once a month or less)

- 3-Sometimes (more than once a month, up to twice a week)

- 4-Often (more than twice a week)

- 5-Everyday

3. How often do you desire to engage in sexual activity?

- 1-Never

- 2-Rarely (once a month or less)

- 3-Sometimes (more than once a month, up to twice a week)

- 4-Often (more than twice a week)

- 5-Everyday

4. How frequently do you engage in sexual thoughts (thinking about, sexual fantasies) now?

- 1-Never

- 2-Rarely (once a month or less)

- 3-Sometimes (more than a month, up to twice a week)

- 4-Often (more than twice a week)

- 5-Everyday

5. Do you enjoy books, movies, music or artwork with sexual content?

- 1-Never

- 2-Rarely (once a month or less)

- 3-Sometimes (more than a month, up to twice a week)

- 4-Often (more than twice a week)

- 5-Everyday

6. How much pleasure or enjoyment do you get from thinking about and fantasizing about sex?

- 1-No enjoyment or pleasure

- 2-Little enjoyment or pleasure

- 3-Some enjoyment or pleasure

- 4-Much enjoyment or pleasure

- 5-Great enjoyment or pleasure

7. How often do have an erection related or unrelated to sexual activity

- 1-Never

- 2-Rarely (once a month or less) 
- 3-Sometimes (more than once a month, up to twice a week)

- 4-Often (more than twice a week)

- 5-Everyday

8. Do you get an erection easily?

- 1-Never

- 2-Rarely (much less than half the time)

- 3-Sometimes (about half the time)

- 4-Often (much more than half the time)

- 5-Always

9. Are you able to maintain an erection?

- 1-Never

- 2-Rarely (much less than half the time)

- 3-Sometimes (about half the time)

- 4-Often (much more than half the time)

- 5-Always

10. How often do you experience painful, prolonged erection?

- 1-Never

- 2-Rarely (once a month or less)

- 3-Sometimes (more than once a month, up to twice a week)

- 4-Often (more than twice a week)

- 5-Everyday

11. How often do you have an ejaculation?

- 1-Never

- 2-Rarely (once a month or less)

- 3-Sometimes (more than once a month, up to twice a week)

- 4-Often (more than twice a week)

- 5-Everyday

12. Are you able to ejaculate when you want to?

- 1-Never

- 2-Rarely (much less than half the time)

- 3-Sometimes (about half the time)

- 4-Often (much more than half the time)

- 5-Always

13. How much pleasure or enjoyment you get from your orgasm?

- 1-No enjoyment or pleasure

- 2-Little enjoyment or pleasure

- 3-Some enjoyment or pleasure

- 4-Much enjoyment or pleasure

- 5-Great enjoyment or pleasure

14. How often do you have painful orgasm?

- 1-Never

- 2-Rarely (once a month or less)

- 3-Sometimes (more than once a month, up to twice a week)

- 4-Often (more than twice a week)

- 5-Everyday

$=$ Pleasure (item 1$)$

$=$ Desire $/$ frequency (item $2+$ item 3 )

$=$ Desire $/$ interest (item $4+$ item $5+$ item 6 )

$=$ Arousal $/$ excitement (item $7+$ item $8+$ item 9)

$=$ Orgasm $/$ completion (item $11+$ item $12+$ item 13$)$

$=$ Total CSFQ score (item 1 to 14 ).

\section{Changes in Sexual Functioning Questionnaire for Females: (CSFQ-F-C)}

1. Compared with the most enjoyable it has ever been, how enjoyable or pleasurable is your sexual life right now?

- 1-No enjoyment or pleasure

- 2-Little enjoyment or pleasure

- 3-Some enjoyment or pleasure

- 4-Much enjoyment or pleasure

- 5-Great enjoyment or pleasure
2. How frequently do you engage in sexual activity (sexual intercourse, masturbation, etc.) now?

- 1-Never

- 2-Rarely (once a month or less)

- 3-Sometimes (more than once a month, up to twice a week)

- 4-Often (more than twice a week)

- 5-Everyday

3. How often do you desire to engage in sexual activity?

- 1-Never

- 2-Rarely (once a month or less)

- 3-Sometimes (more than once a month, up to twice a week)

- 4-Often (more than twice a week)

- 5-Everyday

4. How frequently do you engage in sexual thoughts (thinking about, sexual fantasies) now?

- 1-Never

- 2-Rarely (once a month or less)

- 3-Sometimes (more than a month, up to twice a week)

- 4-Often (more than twice a week)

- 5-Everyday

5. Do you enjoy books, movies, music or artwork with sexual content?

- 1-Never

- 2-Rarely (once a month or less)

- 3-Sometimes (more than a month, up to twice a week)

- 4-Often (more than twice a week)

- 5-Everyday

6. How much pleasure or enjoyment do you get from thinking about and fantasizing about sex?

- 1-No enjoyment or pleasure

- 2-Little enjoyment or pleasure

- 3-Some enjoyment or pleasure

- 4-Much enjoyment or pleasure

- 5-Great enjoyment or pleasure

7. How often do you become sexually aroused?

- 1-Never

- 2-Rarely (once a month or less)

- 3-Sometimes (more than once a month, up to twice a week)

- 4-Often (more than twice a week)

- 5-Everyday

8. Are you easily aroused?

- 1-Never

- 2-Rarely (much less than half the time)

- 3-Sometimes (about half the time)

- 4-Often (much more than half the time)

- 5-Always

9. Do you have adequate vaginal lubrication during sexual activity?

- 1-Never

- 2-Rarely (much less than half the time)

- 3-Sometimes (about half the time)

- 4-Often (much more than half the time)

- 5-Always

10. How often do you become aroused and then lose interest?

- 5-Never

- 4-Rarely (much less than half the time)

- 3-Sometimes (about half the time)

- 2-Often (much more than half the time)

- 1-Always

11. How do you experience an orgasm?

- 1-Never

- 2-Rarely (much less than half the time)

- 3-Sometimes (about half the time)

- 4-Often (much more than half the time)

- 5-Always 
12. Are you able to have an orgasm when you want to?

- 1-Never

- 2-Rarely (much less than half the time)

- 3-Sometimes (about half the time)

- 4-Often (much more than half the time)

- 5-Always

13. How much pleasure or enjoyment do you get from your orgasm?

- 1-No enjoyment or pleasure

- 2-Little enjoyment or pleasure

- 3-Some enjoyment or pleasure

- 4-Much enjoyment or pleasure

- 5-Great enjoyment or pleasure

14. How often do you have painful orgasm?

- 5-Never

- 4-Rarely (once a month or less)

- 3-Sometimes (more than once a month, up to twice a week)

- 2-Often (more than twice a week)

- 1-Everyday

= Pleasure (item 1$)$

$=$ Desire $/$ frequency (item $2+$ item 3$)$

$=$ Desire $/$ interest (item $4+$ item $5+$ item 6$)$

$=$ Arousal $/$ excitement (item $7+$ item $8+$ item 9 )

$=$ Orgasm $/$ completion (item $11+$ item $12+$ item 13 )

$=$ Total CSFQ Score (item 1 to 14)

To calculate the total CSFQ score, add up the values of the responses of all the 14 items. To calculate subscale scores, add up the values for only the items that correspond to a particular subscale (see shaded box on front side) to determine if sexual dysfunction is present, refer to a gender-specific scoring protocols below:

\section{Scoring for CSFQ-F-C (Female clinical version)}

If the female patient obtains a score at or below the following cut-off points* on any of these scale, it is indicative of sexual dysfunction

Total CSFQ score

Sexual desire/frequency score:

Sexual desire/interest:

Sexual pleasure:

Sexual arousal/excitement:

Sexual orgasm/completion:

\section{Scoring for CSFQ-M-C (Male clinical version)}

If the male patient obtains a score at o below the following cut-off points* on any of these scale, it is indicative of sexual dysfunction
Total CSFQ score

Sexual desire/frequency score:

Sexual desire/interest:

Sexual pleasure:

Sexual arousal/excitement:

Sexual orgasm/completion:
47.0 (range: 14 to 70 )

8.0 (range: 2 to 10 )

11.0 (range: 3 to 15$)$

13.0 (range: 3 to 15 )

13.0 (range: 3 to 15 )
4.0 (range: 1 to 5 ) 